\title{
Neoadjuvant Chemotherapy with S-1 and Oxaliplatin followed by Laparoscopic D2 Gastrectomy for Clinical Stage III Gastric Cancer: Primary Results of Phase II nacG-SOX130 Study
}

Sayuri Konishi ${ }^{1 *}$, Dai Manaka ${ }^{1}$, Hideo An ${ }^{1}$, Kiyotaka Kawaguchi ${ }^{1}$, Shinya Hamasu ${ }^{1}$, Masashi Fushitani ${ }^{1}$, Takano Ota ${ }^{1}$, Michina Morioka ${ }^{1}$, Yusuke Okamura ${ }^{1}$, Naoya Sasaki ${ }^{1}$, Ryuta Nishitai ${ }^{1}$, Kensuke Kurihara ${ }^{2}$, Toshihide Yamaoka ${ }^{2}$, Shigehiko Fujii ${ }^{3}$, Toshihiro Kusaka ${ }^{3}$ and Shinsuke Shibuya ${ }^{4}$

${ }^{1}$ Department of Surgery, Gastrointestinal Center, Kyoto Katsura Hospital, Japan

${ }^{2}$ Department of Radiology, Kyoto Katsura Hospital, Japan

${ }^{3}$ Department of Gastroenterology, Gastrointestinal Center, Kyoto Katsura Hospital, Japan

${ }^{4}$ Department of Pathology, Kyoto Katsura Hospital, Japan

${ }^{*}$ Corresponding author: Sayuri Konish, 17, Hirao-cho, Yamada, Nishikyo-ku, Kyoto, 615-8256, Japan; Tel: 81-75-391-5811; Fax: 81-75-381-0090; E-mail: sayurik0713@gmail.com

Received: August 20, 2021; Accepted: August 25, 2021; Published: September 06, 2021

\begin{abstract}
Background: In spite of modern multimodal strategies, the prognosis of Stage III gastric cancer is still insufficient. We conducted a phase II study to evaluate the safety and efficacy of neoadjuvant chemotherapy with S-1 and oxaliplatin for clinical Stage III gastric cancer.

Methods: Patients with clinical Stage III gastric adenocarcinoma were treated with two-cycles of nacG-SOX130 (S-1 80 mg/m2 daily for 2 weeks, oxaliplatin $130 \mathrm{mg} / \mathrm{m} 2$ on day 1, every 3 weeks), followed by laparoscopic D2 gastrectomy. The primary endpoint was the clinical response rate. The planned enrollment was 36 , which was calculated based on an expected response rate of $70 \%$ and a threshold response rate of $56 \%$, with a one-sided alpha of $5 \%$ and a power of $90 \%$.

Results: Between January 2016 and February 2019, 36 patients were enrolled, of whom 34 were assessed for efficacy. R0 resection rate was 97.1\% (33/34). The clinical response rate was $73.5 \%(25 / 34,95 \% \mathrm{CI} 58.70-88.36, \mathrm{p}=0.025)$ and the pathological response rate was $58.8 \%(20 / 34)$. The most common toxicities during neoadjuvant chemotherapy were grade $1 / 2$ neutropenia $(58.8 \%)$ and grade $1 / 2$ peripheral sensory neuropathy $(52.9 \%)$. The grade 3 surgical morbidity was $8.8 \%$ (3/34). Treatment-related death and operative mortality were not observed.
\end{abstract}

Conclusions: nacG-SOX130 was feasible and resulted in encouraging response rates without compromising surgery. nacG-SOX130 would be a promising option of neoadjuvant chemotherapy for clinical Stage III gastric cancer.

Keywords: Neoadjuvant chemotherapy, Laparoscopic surgery, SOX, Gastric cancer

\section{Introduction}

Over the past decade, multimodal treatments have improved the prognosis of resectable gastric cancer. High quality surgery, which should be precise in lymph node dissections and, at the same time, less invasive for quality of life, is also critical for better survival [1]. However, gastric cancer is still one of the leading causes of cancerrelated deaths worldwide, and there is an unmet need for a newer strategy [2]. Radical surgery is an essential step of the treatment. In Asian countries, it is followed by adjuvant chemotherapy according to the pathological status of the tumor. 1-year S-1 monotherapy or 6-month oxaliplatin-combined doublet therapy is widely used in Stage II/III gastric cancer [3,4]. Even with these established strategies, prognosis still remains poor in some patients with high-risk features of recurrence, i.e. Stage III. In the ACTS-GC study, which tested adjuvant S-1 [5], the 5-year overall survival (OS) in Stage II patients was $84.2 \%$ (hazard ratio (HR) $0.509,95 \%$ confidence interval (CI): 0.338-0.765). In contrast, in Stage IIIA and IIIB, the 5-year OS were 67.1\% (HR 0.708, 95\%CI: 0.510-0.983) and 50.2\% (HR 0.791, 95\%CI: $0.520-1.205)$, respectively, which could not be satisfactory as compared with the results of Stage II patients. In the CLASSIC trial, which tested adjuvant capecitabine plus oxaliplatin [6], the 5-year OS in Stage II, IIIA and IIIB were $88 \%$ (HR 0.54, 95\%CI: 0.34-0.87), 70\% (HR 0.75, 95\%CI: $0.52-1.10$ ) and 66\% (HR 0.67, 95\%CI: 0.39-1.13), respectively, which showed the similar trend of OS reduction as seen in the ACTSGC study. Even with a newer adjuvant regimen of S-1 plus docetaxel in Stage III gastric cancer in the JACCRO GC-07 trial, similar reduction of relapse-free survival was observed as the cancer stage progressed, 
and more than half of stage IIIC patients recurred after surgery [7]. Poor compliance in a post-operative phase is one of the reasons for worse outcomes. Not only the surgical complications, but eating disorders and subsequent body weight loss might have substantial impacts on continuity of every treatment [8]. From this standpoint, chemotherapy before surgery, namely neoadjuvant chemotherapy, is a promising option, especially when more intensive and therefore more toxic combined regimens including platinum compounds are considered. Although relative advantages of a preoperative time period are now well understood, some clinical hesitations for neoadjuvant chemotherapy still remain. The positive results of two pivotal studies in perioperative settings, the MAGIC trial [9] and the ACCORD07/ FFCD 9703 trial [10], could not be translated into the current clinical situations because surgical procedures in these studies were not reached to the today's standards of curative intent D2 gastrectomy. Furthermore, it is a well-known fact that racial and ethnic disparities widely exist in the world of gastric cancer [11]. Therefore, in the planning of a new neoadjuvant study in gastric cancer, we should set up a planning with the latest, non-invasive radical D2 gastrectomy, and arrange the experimental regimen suitable for each country. In this phase II study, we evaluated the efficacy and toxicity of neoadjuvant chemotherapy with S-1 plus oxaliplatin for clinical Stage III gastric cancer patients who underwent laparoscopic radical D2 gastrectomy.

\section{Methods}

\section{Study Design}

nacG-SOX130 study was planned as a prospective, singleinstitution, investigator-initiated phase II trial at Kyoto Katsura Hospital, Kyoto, Japan and was conducted in accordance with the Declaration of Helsinki as well as the Japanese Ethical Guidelines for Clinical Studies. The study was approved by the Institutional Review Board and was registered in the University Hospital Medical Information Network Clinical Trials Registry (http://www.umin.ac.jp/ ctr/) as UMIN000036139.

\section{Eligibility Criteria}

The eligibility criteria were; (1) histologically proven and clinically resectable gastric adenocarcinoma; (2) clinical T3-4/N1-3M0 disease: $\mathrm{T}$ and $\mathrm{N}$ stages were determined by computed tomography (CT) based on the 7th UICC/AJCC TNM classification. Positive lymph node was defined as that with a long axis diameter $>8 \mathrm{~mm}$ or a short axis diameter $\geq 6 \mathrm{~mm}$; (3) an age of over 20; (4) an Eastern Cooperative Oncology Group performance status 0-1; (5) no history of prior chemotherapy, radiotherapy, or surgery for gastric cancer; (6) an adequate oral intake without intestinal obstruction; (7) adequate hepatic, renal, cardio-respiratory and bone marrow functions; (8) a written informed consent. The exclusion criteria were; (1) synchronous or metachronous (within 5 years) malignancy other than carcinoma in situ; (2) pulmonary fibrosis, interstitial pneumonitis, bowel obstruction; (3) pregnant or breastfeeding women; (4) active infections; (5) severe mental disease. Before entry in this study, staging laparoscopy was required to exclude peritoneal dissemination. Chest radiography, contrast-enhanced thoracic/abdominal/pelvic
CT and upper gastrointestinal tract endoscopy were conducted as a pretreatment workup.

\section{Treatment Schedule}

Patients were treated with two-cycles of neoadjuvant chemotherapy (nacG-SOX130: S-1 $80 \mathrm{mg} / \mathrm{m}^{2}$ daily for 2 weeks, oxaliplatin $130 \mathrm{mg}$ / $\mathrm{m}^{2}$ on day 1 , every 3 weeks), followed by surgery. Surgery was planned 4-6 weeks after the end of neoadjuvant chemotherapy. Toxicity was assessed according to the Common Terminology Criteria for Adverse Events, version 4.0. The subsequent chemotherapy cycle was delayed until patient recovery for those with severe adverse events. After the second cycle of nacG-SOX130, efficacy was evaluated on the basis of CT findings, tumor marker levels and the upper gastrointestinal endoscopic examination.

\section{Surgery}

Patients underwent surgery between 4 and 6 weeks after the last administration of S-1 if R0 resection was considered possible on the findings of imaging studies and laboratory data. All patients underwent gastrectomy laparoscopically. After placing laparoscopic ports, intraperitoneal washing cytology specimens were sampled first of all to investigate peritoneal dissemination. If cytology was negative, R0 resection was attempted by distal or total laparoscopic gastrectomy with D2 lymphadenectomy according to the Japanese Gastric Cancer Treatment Guideline 2014 (ver. 4) [12]. Involved adjacent organs, if any, were removed to achieve R0 resection. If R0 resection was considered impossible, the protocol treatment was terminated.

\section{Endpoints}

The primary endpoint of this trial was the clinical response rate (cRR), which was calculated by a sum of uni-dimensional measurements of short axis of positive lymph nodes: complete response (CR), disappearance of positive lymph nodes; partial response (PR), at least a $30 \%$ decrease; progressive disease $(\mathrm{PD})$, at least a $20 \%$ increase or appearance of new lesions; stable disease (SD), non-PR and non-PD. Objective responses were evaluated by two experienced physicians who were not informed of the results of each treatment. Concordance between two physicians was evaluated with contingency tables and by Cohen's kappa coefficient. The average value was generated as a representative one for response. The secondary endpoints were R0 resection rate, pathological response rate ( $\mathrm{pRR}$ ), dose intensity of neoadjuvant chemotherapy, toxicities, 3-year relapsefree survival (RFS) and overall survival (OS) from the registration. The pathological response was graded by the institutional pathologists according to the Japanese classification of gastric carcinoma [13]: grade 1a, the degeneration area was less than one-third of the tumor; grade $1 \mathrm{~b}$, more than one-third and less than two-thirds; grade $2 \mathrm{a}$, more than two-thirds but $<90 \%$; grade 2 b, more than $90 \%$ but $<100 \%$; grade 3 , no residual tumor. In this study, the pathological response was defined as grade $1 \mathrm{~b}$ to grade 3 responses. All enrolled patients were followed for 5 years. Physical and blood examinations were conducted every 3 months for the first 3 years and every 6 months for the last 2 years. Abdominal CT was performed at least every 6 months. 


\section{Statistical Considerations}

S-1 plus cisplatin (SP) is a standard treatment for advanced/ recurrent gastric cancer. The cRR of SP was 54\% (95\% CI: 46-62) in the SPIRITS study. On the other hand, the cRR of S-1 plus oxaliplatin was $55.7 \%$ (95\% CI: 50-62) in the G-SOX phase III study. Based on the results of these two studies, the threshold response rate in this trial was set at $56 \%$. The expected response rate was set at $70 \%$. Assuming that a one-tailed score test was performed with an $\alpha$ of $0.05,33$ patients were needed to ensure a statistical power of $90 \%$. Planned enrollment was 36 subjects.

\section{Postoperative Chemotherapy}

S-1 monotherapy was started within 42 days after surgery if R0 resection was achieved pathologically. A 6-week cycle consisting of 4 weeks of oral administration of S-1 at a dosage of $40 \mathrm{mg} / \mathrm{m}^{2}$ twice daily followed by 2 weeks rest was repeated during the first postoperative year. If S-1 therapy was not started within 3 months after surgery for any reason, the protocol treatment was terminated. The protocol treatment was completed when a patient finished postoperative chemotherapy. After completion of the protocol, no further treatment was given until tumor recurrence.

\section{Results}

\section{Patient Characteristics}

From January 2016 to February 2019, staging laparoscopy was performed in 43 consecutive candidates, and seven patients were excluded; three with macroscopic peritoneal disseminations and four with positive cytology. Finally, 36 patients were enrolled, of whom two patients were excluded; one with emergency surgery for inguinal herniation, and the other with patient's withdrawal. Accordingly, 34 patients were assessed for efficacy (Figure 1). There were 28 males and 6 females with a median age of 71.5 years (Table 1 ).
Table 1: Patient and tumor characteristics $(\mathrm{n}=34)$.

\begin{tabular}{|c|c|c|c|}
\hline & & Values & \\
\hline Age (years) ${ }^{\mathrm{a}}$ & & 71.5 & $(40-80)$ \\
\hline \multirow[t]{2}{*}{ Sex } & Male & 28 & $(82 \%)$ \\
\hline & Female & 6 & $(18 \%)$ \\
\hline \multirow[t]{2}{*}{ ECOG PS } & 0 & 34 & $(100 \%)$ \\
\hline & 1 & 0 & $(0 \%)$ \\
\hline \multirow[t]{4}{*}{ Tumor location } & EGJ or cardia & 4 & $(12 \%)$ \\
\hline & Body & 4 & $(12 \%)$ \\
\hline & Antrum, Pylorus & 19 & $(56 \%)$ \\
\hline & Diffuse or multiple & 7 & $(21 \%)$ \\
\hline \multirow[t]{2}{*}{ Histology } & Differentiated & 19 & $(56 \%)$ \\
\hline & Undifferentiated & 15 & $(44 \%)$ \\
\hline \multirow[t]{5}{*}{ Macroscopic type } & 1 & 0 & $(0 \%)$ \\
\hline & 2 & 17 & $(50 \%)$ \\
\hline & 3 & 10 & $(29 \%)$ \\
\hline & 4 & 3 & $(9 \%)$ \\
\hline & 5 & 4 & $(12 \%)$ \\
\hline \multirow[t]{5}{*}{ Tumor depth } & cT0 & 0 & $(0 \%)$ \\
\hline & cT1a, cT1b & 0 & $(0 \%)$ \\
\hline & $\mathrm{cT} 2$ & 0 & $(0 \%)$ \\
\hline & cT3 & 12 & $(35 \%)$ \\
\hline & cT4a, cT4b & 22 & $(65 \%)$ \\
\hline \multirow[t]{4}{*}{ Lymph node metastasis } & cN0 & 0 & $(0 \%)$ \\
\hline & $\mathrm{cN} 1$ & 1 & $(35 \%)$ \\
\hline & $\mathrm{cN} 2$ & 19 & $(56 \%)$ \\
\hline & $\mathrm{cN} 3 \mathrm{a}, \mathrm{cN} 3 \mathrm{~b}$ & 10 & $(29 \%)$ \\
\hline \multirow[t]{2}{*}{ M category } & cM0 & 34 & $(100 \%)$ \\
\hline & cM1 & 0 & $(0 \%)$ \\
\hline
\end{tabular}

TNM categories are based on 14th Japanese classification of gastric carcinoma (corresponding to the third English edition) ECOG Eastern Cooperative Oncology Group ${ }^{a}$ The median is given, with the range in parentheses.

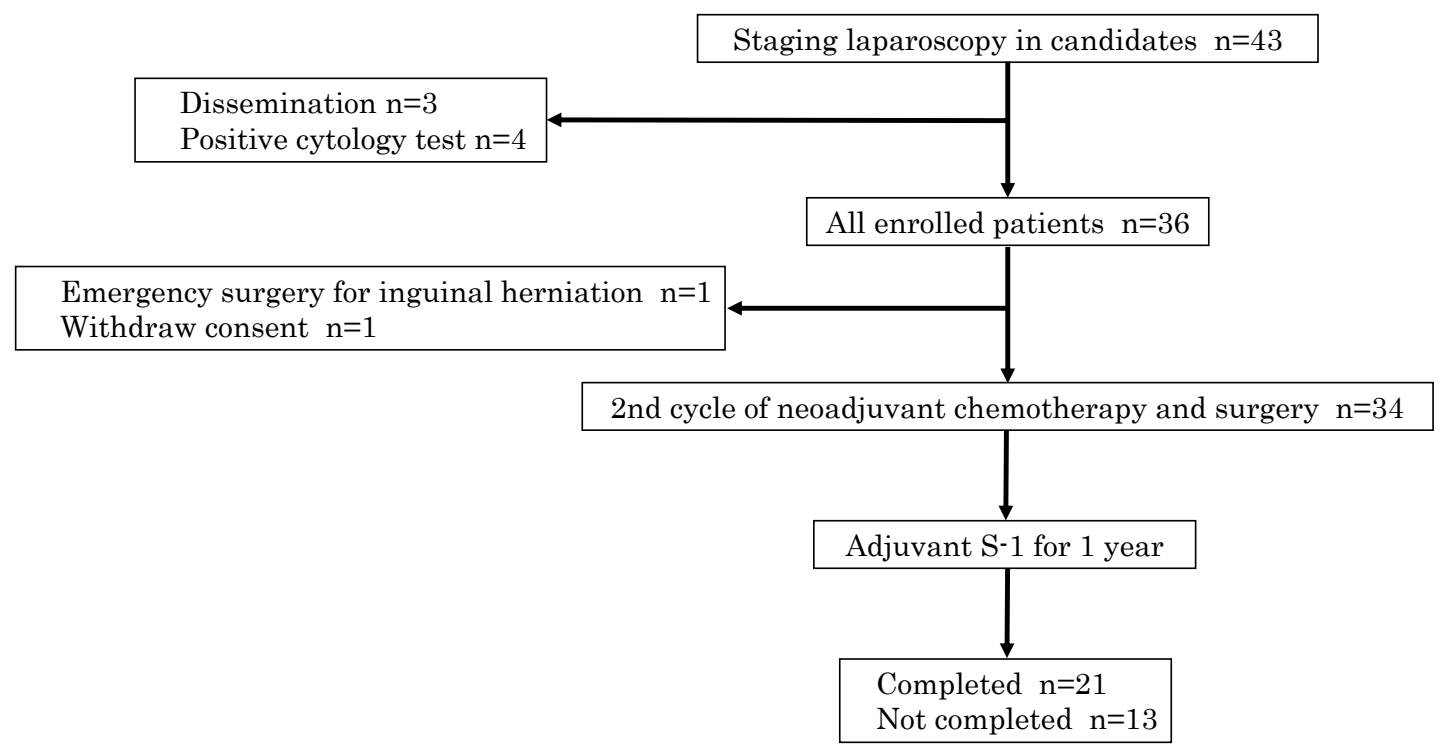

Figure 1: Patient flow chart. Staging laparoscopy was performed in 43 candidates, and seven patients were excluded. Finally 36 patients were enrolled, of whom 34 were assessed for efficacy. Completion rate of protocol treatment was $61.8 \%(21 / 34)$. 


\section{Neoadjuvant Chemotherapy and Toxicities}

The completion rate of two cycles of nacG-SOX130 was $100 \%$. Relative dose intensity was $89.1 \%$ in S-1 (95\%CI: 85.0-93.2) and $97.3 \%$ in oxaliplatin (95\%CI: 95.2-99.4). The most frequent hematological and non-hematological toxicities were neutropenia (Grade1; 35.3\%, Grade2; 23.5\%, Grade3; 0\%) and peripheral sensory neuropathy (Grade1; 50\%, Grade2; 2.9\%, Grade3; 0\%), respectively. No treatmentrelated death was observed (Table 2).

\section{Clinical and Pathological Responses}

In terms of interobserver agreement on clinical responses, substantial concordance was shown by Cohen's kappa coefficient of 0.582 (95\%CI: $0.2934-0.8706, \mathrm{p}<0.001$ ). There were no complete responses, partial responses in 25 patients $(73.5 \%)$ and stable disease in 9 patients (26.5\%). No progressive disease was observed (Table 3 ). The cRR was $73.5 \%$ (95\%CI: 58.70-88.36), and the null hypothesis was rejected (one-sided $p=0.025)$. The pRR was $58.8 \%$ (20/34), including 3 pathological complete response cases (8.8\%). 17 patients were revealed as ypN0 status (50\%) (Table 4). In case of applying $10 \%$ as a cutoff for residual tumor (Grade $2 \mathrm{~b} / 3$ ), this conditional pRR was $26.4 \%(9 / 34)$.

\section{Surgical Findings}

34 patients underwent D2 gastrectomy (100\%). No patients had peritoneal dissemination at the planned operation. Concomitant splenectomy was performed in one patient. R0 resection rate was 97.1\% (33/34) with one R1 case who had a positive proximal margin. 31 patients had no postoperative complication more than G1 (91.2\%). 3 patients had G3 complications (8.8\%); one with postoperative bleeding and two with intra-abdominal abscess. No operative mortality was observed (Table 5).

\section{Postoperative Chemotherapy}

Of 34 patients, 32 subsequently began postoperative chemotherapy as a protocol treatment $(94.1 \%)$. Postoperative chemotherapy was not started in the remaining 2 patients due to renal dysfunction and postoperative anastomotic stenosis, respectively. Of the 32 patients who started postoperative chemotherapy, 21 completed postoperative S-1 therapy for 1 year $(65.6 \%)$. Therefore, the completion rate of the protocol treatment comprising neoadjuvant nac-GSOX130, surgical resection, and postoperative $S 1$ was $61.8 \%(21 / 34)$.

\section{Survival after Resection}

RFS and OS were assessed for 34 patients. After a median followup of 38.05 months, the 3-year RFS rate was $65.7 \%$ (38.23\% of RFS events, $95 \%$ CI: $46.5 \%$ to 79.5 ) and the 3 -year OS rate was $64.3 \%$ (35.29\% of OS events, $95 \%$ CI: $44.6 \%$ to 78.6 ) (Figure 2).

Table 2: Adverse events during chemotherapy $(n=34)$.

\begin{tabular}{|c|c|c|c|c|c|c|c|}
\hline & Any Grade ${ }^{a}$ & $(\%)$ & Grade 1 & $(\%)$ & Grade 2 & $(\%)$ & Grade 3 \\
\hline \multicolumn{8}{|l|}{ Hematological toxicity } \\
\hline Leukopenia & 8 & $(23.5)$ & 4 & $(11.8)$ & 4 & $(11.8)$ & 0 \\
\hline Neutropenia & 20 & $(58.8)$ & 12 & $(35.3)$ & 8 & $(23.5)$ & 0 \\
\hline Anemia & 27 & $(79.4)$ & 21 & $(61.8)$ & 6 & $(17.6)$ & 0 \\
\hline Thrombocytopenia & 14 & $(41.2)$ & 13 & $(38.2)$ & 1 & (2.9) & 0 \\
\hline \multicolumn{8}{|c|}{ Non-hematological toxicity } \\
\hline Peripheral neuropathy & 18 & $(52.9)$ & 17 & $(50)$ & 1 & $(2.9)$ & 0 \\
\hline General malaise & 2 & $(5.9)$ & 2 & $(5.9)$ & 0 & $(0)$ & 0 \\
\hline Fever & 2 & (5.9) & 2 & (5.9) & 0 & $(0)$ & 0 \\
\hline Diarrhea & 2 & (5.9) & 1 & $(2.9)$ & 1 & $(2.9)$ & 0 \\
\hline Anorexia & 6 & $(17.6)$ & 6 & $(17.6)$ & 0 & $(0)$ & 0 \\
\hline Constipation & 2 & (5.9) & 2 & (5.9) & 0 & $(0)$ & 0 \\
\hline Eczema & 2 & (5.9) & 2 & (5.9) & 0 & $(0)$ & 0 \\
\hline Dry eye & 1 & (2.9) & 1 & $(2.9)$ & 0 & $(0)$ & 0 \\
\hline
\end{tabular}

a National Cancer Institute Common Terminology Criteria for Adverse Events version 4.0 (CTCAE ver. 4.0).

Table 3: Clinical response $(n=34)$.

\begin{tabular}{lcc}
\hline Response & Values & \% (95\% CI $)$ \\
\hline CR & 0 \\
PR & 25 & 0 \\
SD & 9 \\
PD & 0 \\
Overall response rate $(\mathrm{CR}+\mathrm{PR})$ & 25 \\
Disease control rate $(\mathrm{CR}+\mathrm{PR}+\mathrm{SD})$ & 34 & 0 \\
\hline
\end{tabular}

CR complete response, $\mathrm{PR}$ partial response, $\mathrm{SD}$ stable disease, $\mathrm{PD}$ progressive disease. 


\section{Discussion}

In this phase II study, we demonstrated that neoadjuvant chemotherapy with nacG-SOX130 had a significantly better cRR with acceptable safety and feasibility for clinical Stage III gastric cancer (73.5\%, 95\%CI: 58.70-88.36, $\mathrm{p}=0.025)$. Secondary endpoints also

\begin{tabular}{|c|c|c|c|}
\hline & Values & $\%$ & $(95 \% \mathrm{CI})$ \\
\hline \multicolumn{4}{|l|}{ Tumor depth } \\
\hline урТ0 & 3 & 8.8 & \\
\hline ypT1a, ypT1b & 2 & 5.9 & \\
\hline ypT2 & 6 & 17.6 & \\
\hline ypT3 & 16 & 47.1 & \\
\hline урТ4a, урT4b & 7 & 20.6 & \\
\hline \multicolumn{4}{|l|}{ Lymph node metastasis } \\
\hline ypN0 & 17 & 50 & \\
\hline ypN1 & 9 & 26.5 & \\
\hline ypN2 & 4 & 11.8 & \\
\hline ypN3a, ypN3b & 4 & 11.8 & \\
\hline \multicolumn{4}{|l|}{ Resection } \\
\hline R0 & 33 & 97.1 & \\
\hline $\mathrm{R} 1$ & 1 & 2.9 & \\
\hline \multicolumn{4}{|l|}{ Pathological response } \\
\hline Grade 0 & 4 & 11.8 & \\
\hline Grade la & 10 & 29.4 & \\
\hline Grade $1 b$ & 3 & 8.8 & \\
\hline Grade 2a & 8 & 23.5 & \\
\hline Grade $2 b$ & 6 & 17.6 & \\
\hline Grade 3 & 3 & 8.8 & \\
\hline $\begin{array}{l}\text { Pathological response ra } \\
\quad \text { (grade } 1 \mathrm{~b}, 2 \mathrm{a}, 2 \mathrm{~b}, 3)\end{array}$ & 20 & 58.8 & $(42.3-75.3)$ \\
\hline
\end{tabular}

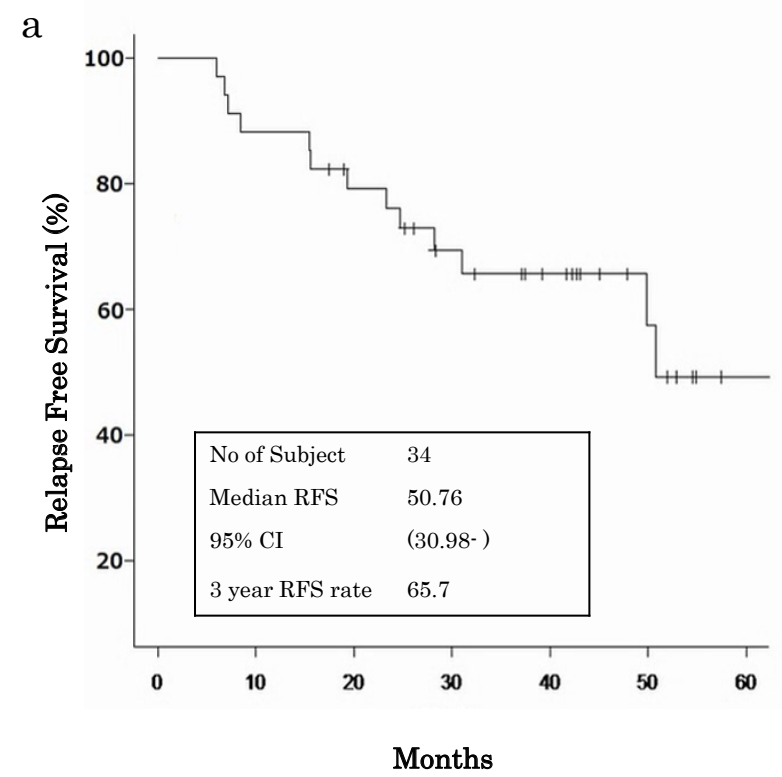

showed favorable results, in which pRR was $58.8 \%$ (20/34), including 3 pathological complete response cases (8.8\%) and the 3-year RFS rate was $65.7 \%$ (95\% CI: $46.5 \%$ to 79.5 ) and the 3 -year OS rate was $64.3 \%$ ( $95 \%$ CI: $44.6 \%$ to 78.6 ). These results were encouraging for selecting the next candidate of neoadjuvant treatment in multimodal strategy for clinical Stage III gastric cancer.

Although surgery is the main-stay of treatment of gastric cancer, multimodal strategy is needed to further improve the prognosis, especially in the high-risk patients of recurrence, i.e. Stage III [14]. However, in the situation where surgery is very precise with D2 gastrectomy, there are still arguments about necessity of neoadjuvant treatment, because high quality lymphadenectomy might marginalize the contribution of neoadjuvant chemotherapy, which would be otherwise hazardous for subsequent surgery owing to fibrosis and edema induced by chemo-drugs. Furthermore, several negative results of Japan Clinical Oncology Group (JCOG) studies for preoperative chemotherapy using cisplatin for advanced gastric cancer complicated an interpretation whether the regimens using platinum compound were effective or not in the neoadjuvant setting compared to the unresectable, metastatic setting, although these JCOG studies did not

\begin{tabular}{lccccc}
\multicolumn{5}{c}{ Table 5: Surgical complications $(\mathrm{n}=34)}$. \\
\hline Morbidity & Grade 1 $^{\text {a }}$ & Grade 2 & Grade 3a & Grade 3b & \% Grade 3a/b \\
\hline Anastomotic leakage & 0 & 0 & 0 & 0 & 0 \\
Pancreatic fistula & 0 & 0 & 0 & 0 & 0 \\
Pneumonia & 0 & 0 & 0 & 0 & 0 \\
ileus & 0 & 0 & 0 & 0 & 0 \\
Pleural disorder & 0 & 0 & 0 & 0 & 0 \\
Emptying disorder & 0 & 0 & 0 & 0 & 0 \\
Post-operative bleeding & 0 & 0 & 0 & 1 & 2.9 \\
Abdominal infection & 0 & 0 & 2 & 0 & 5.9 \\
\hline
\end{tabular}

${ }^{a}$ National Cancer Institute Common Terminology Criteria for Adverse Events version 4.0 (CTCAE ver. 4.0).

b

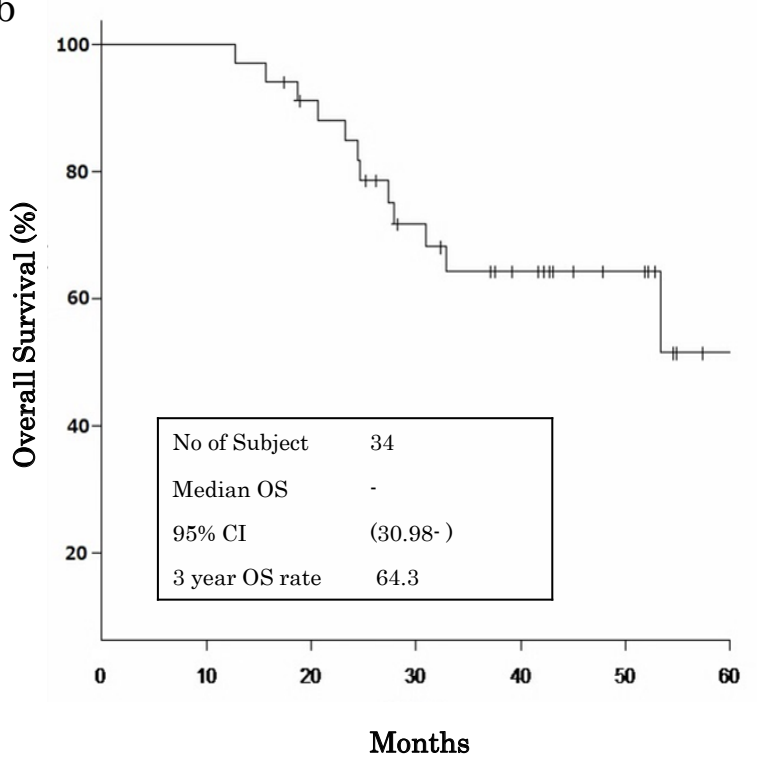

Figure 2: (a) 3 year RFS rate was $65.7 \%$. (b) 3 year OS rate was $64.3 \%$. RFS relapse free survival, OS overall survival, 95 CI $95 \%$ confidence interval. 
exactly target the pure neoadjuvant population, i.e. Stage II/III patients [15-17]. On the other hand, the proof of principle that other platinum, i.e. oxaliplatin, would bring a different outcome from cisplatin has already been shown in pre-clinical and clinical models in gastric cancer. Tan et. al analyzed gene expression profiles for gastric cancer cell lines and identified the intrinsic subtypes which had a favorable response to oxaliplatin instead of cisplatin [18]. In the clinical setting, the AIO-FLOT4 study demonstrated that the replacement of cisplatin to oxaliplatin in combination with epirubicin to docetaxel, did show a higher rate of curative resection $(84 \%$ versus $77 \%, \mathrm{p}=0.01)$, and prolonged OS [HR $0.77(0.63-0.94), p=0.012$ ] [19]. More recently, oxaliplatin-specific signatures based on tumor biology are rigorously sought after in gastric cancer [20]. In order to clarify these confusing understandings about neoadjuvant chemotherapy for resectable gastric cancer, it is necessary to plan a new study targeting the pure neoadjuvant population of Stage III patients with a regimen containing oxaliplatin instead of cisplatin, and also with the latest non-invasive radical D2 surgery.

In the recruitment of candidates in neoadjuvant treatment, exact evaluations of cancer staging should be done before treatment. Nonetheless, there is no explicit consensus for the size criteria for lymph node metastasis. Generally, metastatic lymph nodes are considered to be large. However, metastatic lymph nodes are not necessarily large in size [21], and even more, the majority of metastatic lymph nodes are smaller than $10 \mathrm{~mm}$ [22]. With the technological improvement of diagnostic performance, current CT scanners already have the ability to detect lymph nodes less than $5 \mathrm{~mm}$ in diameter. In the setting of a cut-off value, we should lower the threshold and increase sensitivity to recruit as many patients who need neoadjuvant treatment. Thus, it is still challenging to establish the optimal size criteria for clinical $\mathrm{N}$ status with the trade-off balance of sensitivity and specificity.

The cRR, which we used as a primary endpoint in this study, is expected as an on-treatment marker for personalized treatment [23]. It would be a next challenge to monitor on-treatment efficacy and arrange the scheduled treatment to promote the personalized medicine in neoadjuvant chemotherapy for gastric cancer. The $\mathrm{pRR}$, which was one of the secondary endpoints, was $58.8 \%$ (95\%CI: 76.899.6), and was favorable compared to those in previous studies (48$51 \%)[17,24,25]$. In addition, the conditional pRR of $26.4 \%$ by the threshold of $10 \%$ of the residual tumor was also favorable compared to those of previous study (15.4-19.4\%) [26]. Another thing that we want to highlight here is the high rate of ypN0 status $(17 / 34,50 \%)$. Pathological N0 status is reported to be more linked to better survival than pRR. Achieving ypN0 status was shown as an important hallmark demonstrating the effectiveness of neoadjuvant therapy in gastric cancer [27]. In this context, our result of ypN0 is promising in the future analysis of survival.

The chemotherapy-related adverse events are also critical in the neoadjuvant setting because the safety for subsequent surgery should be warranted. In this study, grade 3 or higher adverse events were not observed both in hematological and non-hematological categories (Table 3). The minimal number of treatment cycles for enough tumor control, which we think to be 2 cycles, might have contributed to the reduction of adverse events. As for peripheral sensory neuropathy induced by oxaliplatin, $94.4 \%$ of the toxicities were Grade 1 (17/18), and only one patient had Grade 2 neuropathy. It is important to note that oxaliplatin has cumulative toxicity, and here again, 2 cycles of oxaliplatin might have resulted in a relatively low rate and grade of neuropathy. So far, treatment duration of neoadjuvant chemotherapy has not been established yet. However, looking at the balance of treatment effects and adverse events demonstrated in this study, it seems to be not always necessary to increase the treatment cycles more than 2, which was also suggested in COMPASS trial [28]. In addition, considering that the median age of patients in this study was relatively high at 71.5 years, safety and tolerability of nacG-SOX130 might be further suggested.

Surgical morbidities were two cases of abdominal infection and one case of postoperative bleeding only (5.9\% and $2.9 \%$, respectively). Anastomotic leakage and pancreatic fistula, which were the most serious complications in gastric cancer surgery, were not observed in this study, suggesting the quality procedures of noninvasive laparoscopic D2 gastrectomy in this study. Since neoadjuvant chemotherapy is premised on surgery, it must always be evaluated in the context of surgical outcomes, and this study demonstrated feasibility of nacG-SOX130 for the subsequent radical surgery.

There are some limitations in this study. First, this trial had a single-arm, phase II design conducted in a single institution with a limited number of patients. Secondly, only the short-term results were analyzed. After the follow-up period will be completed, the efficacy of nacG-SOX130 should be reevaluated in terms of survival.

\section{Conclusion}

In conclusion, nacG-SOX130 in clinical Stage III gastric cancer was feasible and the efficacy results of clinical and pathological responses were encouraging in this high-risk population without impairing curative intent laparoscopic D2 gastrectomy. nacG-SOX130 would be a promising candidate of neoadjuvant treatment for Stage III gastric cancer. With a high rate of ypN0, the future analysis of survival will be expected.

\section{Acknowledgements}

We thank all the patients and families who participated in this trial.

\section{Compliance with Ethical Standards}

\section{Conflict of Interest}

The authors declare that they have no conflict of interest.

\section{Human Rights Statement and Informed Consent}

All procedures followed were in accordance with the ethical standards of the responsible committee on human experimentation (institutional and national) and with the Helsinki Declaration of 1964 and later versions. Informed consent or substitute for it was obtained from all patients for being included in the study.

\section{References}

1. Aggelis V, Cunningham D, Lordick F, Smyth EC (2018) Peri-operative therapy for operable gastroesophageal adenocarcinoma: past, present and future. Annals of Oncology 29: 1377-1385. [crossref]

2. Ferlay J, Colombet M, Soerjomataram I, Mathers C, D M Parkin, et al. (2019) Estimating the global cancer incidence and mortality in 2018. GLOBOCAN sources and methods. International journal of cancer 144: 1941-1953. [crossref] 
3. Japanese Gastric Cancer Association jgca@ koto. kpu-m. ac. Jp. (2020) Japanese gastric cancer treatment guidelines 2018. Gastric Cancer 1-21.

4. Group DW, Panel R (2019) Guideline Committee of the Korean Gastric Cancer Association. Korean practice guideline for gastric cancer 2018: an evidence-based, multi-disciplinary approach. Journal of gastric cancer 19: 1. [crossref]

5. Sasako M, Sakuramoto S, Katai H, Kinoshita T, Hiroshi Furukawa, et al. (2011) Fiveyear outcomes of a randomized phase III trial comparing adjuvant chemotherapy with S-1 versus surgery alone in stage II or III gastric cancer. J clin oncol 29: 43874393. [crossref]

6. Noh SH, Park SR, Yang HK, Chung HC, Ik-Joo Chung, et al. (2014) Adjuvant capecitabine plus oxaliplatin for gastric cancer after D2 gastrectomy (CLASSIC): 5 -year follow-up of an open-label, randomised phase 3 trial. The Lancet Oncology 15: 1389-1396. [crossref]

7. Yoshida K, Kodera Y, Kochi M, Ichikawa W, et al. (2019) Addition of docetaxel to oral fluoropyrimidine improves efficacy in patients with stage III gastric cancer: interim analysis of JACCRO GC-07, a randomized controlled trial. Journal of Clinical Oncology 37: 1296.

8. Kanazawa Y, Yamada T, Kakinuma D, Matsuno K, Fumihiko Ando, et al. (2020) Skeletal Muscle Mass Depletion After Gastrectomy Negatively Affects the Prognosis of Patients With Gastric Cancer. Anticancer Research 40: 4271-4279. [crossref]

9. Cunningham D, Allum WH, Stenning SP, Thompson JN, Marianne Nicolson, et al. (2006) Perioperative chemotherapy versus surgery alone for resectable gastroesophageal cancer. New England Journal of Medicine 355: 11-20. [crossref]

10. Boige V, Pignon J, Saint-Aubert B, Lasser P, et al. (2007) Final results of a randomized trial comparing preoperative 5 -fluorouracil $(\mathrm{F}) /$ cisplatin $(\mathrm{P})$ to surgery alone in adenocarcinoma of stomach and lower esophagus (ASLE): FNLCC ACCORD07FFCD 9703 trial. Journal of Clinical oncology 25: 4510-4510.

11. Merchant SJ, Li L, Kim J (2014) Racial and ethnic disparities in gastric cancer outcomes: more important than surgical technique? World Journal of Gastroenterology 20: 11546. [crossref]

12. Japanese Gastric Cancer Association, et al. (2017) Japanese gastric cancer treatment guidelines 2014 (ver. 4). Gastric cancer 20: 1-19. [crossref]

13. Japanese Gastric Cancer Association jgca@ koto. kpu-m. ac. jp. (2011) Japanese classification of gastric carcinoma: 3rd English edition. Gastric cancer 14: 101-112. [crossref]

14. Smyth EC, Nilsson M, Grabsch HI, van Grieken NC, et al. (2020) Gastric cancer. The Lancet 396: 635-648.

15. Yoshikawa T (2007) A phase II study of preoperative chemotherapy with irinotecan and cisplatin followed by gastrectomy with D3 dissection for gastric cancer with extensive lymph node metastasis: Final results of JCOG0001. In Proc Am Soc Clin Oncol 40.

16. Ito S, Sano T, Mizusawa J, Takahari D, et al. (2017) A phase II study of preoperative chemotherapy with docetaxel, cisplatin, and S-1 followed by gastrectomy with D2 plus para-aortic lymph node dissection for gastric cancer with extensive lymph node metastasis: JCOG1002. Gastric Cancer 20: 322-331.
17. Iwasaki Y, Terashima M, Mizusawa J, Katayama H, et al. (2020) Gastrectomy with or without neoadjuvant S-1 plus cisplatin for type 4 or large type 3 gastric cancer (JCOG0501): an open-label, phase 3, randomized controlled trial. Gastric Cancer 1-11. [crossref]

18. Tan IB, Ivanova $\mathrm{T}$, Lim $\mathrm{KH}$, Ong CW, et al. (2011) Intrinsic subtypes of gastric cancer, based on gene expression pattern, predict survival and respond differently to chemotherapy. Gastroenterology 141: 476-485. [crossref]

19. Al-Batran SE, Homann N, Schmalenberg H, Kopp HG, et al. (2017) Perioperative chemotherapy with docetaxel, oxaliplatin, and fluorouracil/leucovorin (FLOT) versus epirubicin, cisplatin, and fluorouracil or capecitabine (ECF/ECX) for resectable gastric or gastroesophageal junction (GEJ) adenocarcinoma (FLOT4-AIO): A multicenter, randomized phase 3 trial. Journal of clinical oncology 4004. [crossref]

20. Wang Q, Liu X, Chen C, Chen J, et al. (2021) A predictive signature for oxaliplatin and 5 -fluorouracil based chemotherapy in locally advanced gastric cancer. Translational oncology 14: 100901.

21. Noda N, Sasako M, Yamaguchi N, Nakanishi Y (1998) Ignoring small lymph nodes can be a major cause of staging error in gastric cancer. British journal of surgery 85: 831-834.

22. Kim DJ, Kim W (2018) Is lymph node size a reliable factor for estimating lymph node metastasis in early gastric cancer? Journal of gastric cancer 18: 208-208. [crossref]

23. Tarhini A, Kudchadkar RR (2018) Predictive and on-treatment monitoring biomarkers in advanced melanoma: Moving toward personalized medicine. Cancer treatment reviews 71: 8-18. [crossref]

24. Kawashima Y (2008) Phase II study of preoperative neoadjuvant chemotherapy (CX) with S-1 plus cisplatin for gastric cancer (GC) with bulky and/or para-aortic lymph node metastases: A Japan Clinical Oncology Group Study (JCOG0405). In ASCO GI symposium abstract 2008 .

25. Iwasaki Y, Sasako M, Yamamoto S, Nakamura K, et al. (2013) Phase II study of preoperative chemotherapy with S-1 and cisplatin followed by gastrectomy for clinically resectable type 4 and large type 3 gastric cancers (JCOG0210). Journal of surgical oncology 107: 741-745. [crossref]

26. Aoyama T, Nishikawa K, Fujitani K, Ito S, et al. (2017) Early results of a randomized two-by-two factorial phase II trial comparing neoadjuvant chemotherapy with two and four courses of cisplatin/S-1 and docetaxel/cisplatin/S-1 as neoadjuvant chemotherapy for locally advanced gastric cancer. Annals of Oncology 28: 1876-1881. [crossref]

27. Ikoma N, Estrella JS, Hofstetter W, Das P, et al. (2018) Nodal downstaging in gastric cancer patients: promising survival if ypN0 is achieved. Annals of surgical oncology 25: 2012-2017. [crossref]

28. Yoshikawa T, Tanabe K, Nishikawa K, Ito Y, et al. (2014) Induction of a pathological complete response by four courses of neoadjuvant chemotherapy for gastric cancer early results of the randomized phase II COMPASS trial. Annals of surgical oncology 21: 213-219. [crossref]

\section{Citation:}

Konishi S, Manaka D, An H, Kawaguchi K, Hamasu S, et al. (2021) Neoadjuvant Chemotherapy with S-1 and Oxaliplatin followed by Laparoscopic D2 Gastrectomy for Clinical Stage III Gastric Cancer: Primary Results of Phase II nacG-SOX130 Study. Cancer Stud Ther J Volume 6(3): 1-7. 